\title{
Programa de atualização permanente da base contínua 1:250.000, uma visão de futuro
}

\author{
Rafael Balbi Reis* \\ Luiz Antonio Xavier* \\ Maurício Krumbiegel* \\ Alessandra Luiza Gouveia* \\ Renata Curi de Moura E. Nagatomi* \\ Leila Freitas de Oliveira* \\ Marcelo R. de Albuquerque Maranhão*
}

Recebido 30 de maio de 2014; aceito 20 de junho de 2014

\begin{abstract}
This paper has the aim to present the Continual Cartographic Base from Brazil on scale of 1:250.000 - BC250 inside the Permanent Program Updating from IBGE. This program consists in update and maintenance permanent of the whole Brazilian territory, this mean a strategy to subsidize the regions management and also contribute to studies that use environmental politics. These activities of updating require uses of satellite images, like Landsat, CBERS, RapidEye, digital elevation model, information from other institutions and field work. The incorporation of technologies like Geographic Information System and Remote Sensing in the systematic mapping contributed to developing of a base unique and integrated of the whole brazilian territory. The process of construction of BC250 began in 2008, the Brazil was split in parts for cont

ract, and finished this mapping process in August of 2012. The first version of this continual vectorial base is available for consulting on the IBGE website $<$ www.ibge.gov.br> since October of 2013 and in the National Infrastructure Spatial Data (INDE) website <www.inde.gov.br> since December of 2013. This cartographic base is the first one to be produced in Brazil with the new standard of

* Coordenação de Cartografia, Diretoria de Geociências, Instituto Brasileiro de Geografia e Estatística (IBGE), Av. Brasil, ${ }^{\circ}$ 15671, Bloco III, Parada de Lucas, Rio de Janeiro, Brasil. \{rafael.reis, luiz.xavier, mauricio.krumbiegel, alessandra.gouveia, renata.curi, leila.oliveira, marcelo.maranhao\}@ibge.gov.br
\end{abstract}


the Geospatial Data Vectorial Structure (EDGV) that contemplate a new structure of storage of geospatial data for integration with data of INDE.

Key words: remote sensing, geographic information system, cartographic base.

\section{Resumen}

Este trabajo tiene como objetivo presentar la Base Cartográfica Continua de Brasil en la escala de 1:250,000 - BC250; se inserta en el Programa de Actualización Permanente (PAP) del IBGE. Este programa consiste en la actualización y mantenimiento continuo de la cartografía de todo el territorio brasileño a una escala de 1:250.000, con una vista que se trata de un apoyo estratégico para el gobierno en la planificación regional y también contribuye a los estudios sobre las políticas ambientales. Estas actividades incluyen el uso de PAP, foto sensores de los satélites como el Landsat, CBERS, RapidEye, modelos digitales de elevación, las aportaciones de los organismos asociados y los estudios de campo. La incorporación de la tecnología asociada a los Sistemas de Información Geográfica y Sensores Remotos para el mapeo sistemático contribuyó al desarrollo de una sola base integrada en todo Brasil. El proceso de construcción de BC250 se inició en 2008; con Brasil se divide en bloques de la contratación; y la conclusión de la etapa de la cartografía, en agosto de 2012. Una primera versión de la base continua está disponible para consulta en el sitio web de IBGE (www.ibge.gov.br) desde octubre de 2013 y en la página de la Infraestructura Nacional de Datos Espaciales (INDE) desde diciembre de $2013<$ www.inde.gov.br>. Este mapa base es el primero en ser producido en Brasil en la Norma Técnica Especificaciones para los Datos Geoespaciales Estructuración Vectorial (ET-EDGV) que proporciona una nueva estructura para el almacenamiento de datos geoespaciales para la integración de datos en el INDE.

Palabras clave: detección remota, sistema de información geográfica, base cartográfica.

\section{Resumo}

Este trabalho tem como objetivo apresentar a Base Cartográfica Contínua do Brasil na escala de 1:250.000 - BC250, inserido dentro do Programa de Atualização Permanente (PAP) do IBGE. Este programa consiste na atualização e manutenção permanente do mapeamento de todo o território brasileiro na escala de 1:250.000, tendo em vista tratar-se de uma estratégia para subsidiar o governo no planejamento regional e também contribuir para os estudos que envolvem as políticas ambientais. Estas atividades do PAP abrangem o uso de imagens de sensores orbitais, como Landsat, CBERS, RapidEye, modelos digitais de elevação, insumos de órgãos 
parceiros e levantamentos de campo. A incorporação de tecnologias associadas aos Sistemas de Informação Geográfica (SIG) e ao Sensoriamento Remoto (SR) no mapeamento sistemático contribuiu para o desenvolvimento de uma base única $\mathrm{e}$ integrada de todo o território brasileiro. $\mathrm{O}$ processo de construção da $\mathrm{BC} 250$ iniciou-se no ano de 2008, com o Brasil dividido em blocos de contratação, e concluindo-se a etapa de mapeamento em agosto de 2012. A primeira versão da base contínua se encontra disponível para consulta no portal do IBGE $<$ www.ibge.gov.br $>$ desde outubro de 2013 e na página da Infraestrutura Nacional de Dados Espaciais (INDE) desde dezembro de 2013 <www.inde.gov.br>. Esta base cartográfica é a primeira a ser produzida no Brasil no padrão da Especificação Técnica para a Estruturação de Dados Geoespaciais Vetoriais (ET-EDGV), que contempla uma nova estrutura para armazenamento de dados geoespaciais para a integração dos dados na INDE.

Palavras chave: sensoriamento remoto, sistema de informação geográfica, base cartográfica.

\section{Introdução}

A evolução tecnológica tem promovido a popularização das geotecnologias, renovando o interesse da sociedade em reconhecer e reproduzir os fenômenos do espaço geográfico.Tal fato tem incentivado a produção e a disseminação de produtos cartográficos por todo o mundo, bem como seu aprimoramento.

O Brasil é um país de dimensões continentais onde todo e qualquer trabalho que consista em levantar informações sobre o território envolve um quantitativo de pessoal e investimentos consideráveis. Conforme a Tabela 1, o país não apresenta cobertura cartográfica integral nas escalas maiores ou iguais a 1:100.000, sendo que apenas pequenas partes do território estão mapeadas em 1:25.000.

As tecnologias associadas às áreas de Sensoriamento Remoto (SR) e Sistemas de Informações Geográficas (SIG) vêm contribuindo para importantes mudanças na sistemática de trabalho e no levantamento de informações.

Tabela 1

Percentual mapeado do Brasil por escala

\begin{tabular}{lc}
\hline Escalas & Percentual mapeado \\
\hline $1: 1.000 .000$ & $100 \%$ \\
$1: 250.000$ & $100 \%$ \\
$1: 100.000$ & $68 \%$ \\
$1: 50.000$ & $15 \%$ \\
$1: 25.000$ & $1 \%$ \\
\hline
\end{tabular}

Fonte: Adaptado de mapa índice IBGE, 2003. 
Segundo Delou (2006), os SIGs vêm originando uma nova demanda para o mapeamento sistemático terrestre, sendo um fator de pressão a mais para a criação e atualização de bases cartográficas digitais. Além disso, segundo Reis et al. (2005), o emprego de dados gerados a partir de modelos digitais de elevação (MDE), a exemplo do Shuttle Radar to Pography Mission (SRTM, 2010), como fonte de informação altimétrica pode vir a suprir necessidades decorrentes da ausência ou escassez de levantamentos topográficos em muitas regiões do globo terrestre, causadas pela dificuldade de acesso ou pela falta de projetos de mapeamento.

Acompanhando as inovações tecnológicas, em agosto de 2012 o IBGE lançou uma atualização do mapeamento na escala 1:250.000, em formato digital, cobrindo $100 \%$ do território nacional, constituindo a Base Cartográfica Contínua do Brasil na escala 1:250.000. Esta base cartográfica é oriunda da extração de elementos interpretados em imagens de satélite como Landsat e CBERS, representados em feições e com atributos estruturados conforme o padrão estabelecido pela Comissão Nacional de Cartografia (CONCAR). O mapeamento na escala 1:250.000 é importante quando se pensa em demandas de gestão para subsidiar o planejamento regional de projetos que envolvam ou modifiquem o meio ambiente (Reis et al., 2011).

\section{Objetivo}

O Programa de Atualização Permanente da Base Cartográfica Continua do Brasil na escala de 1:250.000 - PAP BC250, objetiva a atualização e manutenção do mapeamento planialtimétrico de todo o território brasileiro nesta escala através do uso de imagens de sensores orbitais, como Landsat, CBERS, RapidEye, modelos digitais de elevação - SRTM, de insumos de órgãos setoriais parceiros, como o Departamento Nacional de Infraestrutura de Transportes (DNIT), Agência Nacional de Águas (ANA), Ministério do Meio Ambiente (MMA), entre outros, além de trabalhos de reambulação realizados em campo.

\section{Área de abrangência}

A área de abrangência é todo território brasileiro, correspondendo a 548 folhas do mapeamento sistemático, tendo sido trabalhada em blocos que seguiram, aproximadamente, a distribuição dos biomas brasileiros ao longo de 3 anos de contratação (Figura 1). Deste total de folhas, 240 corresponderam à atualização do mapeamento existente proveniente do convênio entre o IBGE e o Sistema de Vigilância da Amazônia (SIVAM) (Santos e Oliveira, 2003), que atualizou por imagem de satélite (Landsat), entre os anos de 1998 a 2002, toda área denominada como Amazônia Legal. As 308 folhas restantes do território foram produzidas a partir de um novo mapeamento. 
A avaliação do grau de atualização das cartas existentes foi realizada segundo a metodologia desenvolvida por Maranhão (2005). De modo resumido, tal metodologia leva em consideração alguns indicadores, sendo os principais a data de aquisição das fotografias aéreas, o tamanho da população e sua taxa de crescimento, sendo determinantes para tornar as áreas de certos municípios prioritárias para a elaboração de novos mapeamentos.

Considerando os indicadores citados, identificou-se que 308 folhas deveriam ser refeitas, pois as folhas que originaram esse mapeamento (compiladas das escalas de 1:50.000 e/ou 1:100.000) tinham sido confeccionadas entre as décadas de 60 e 70.

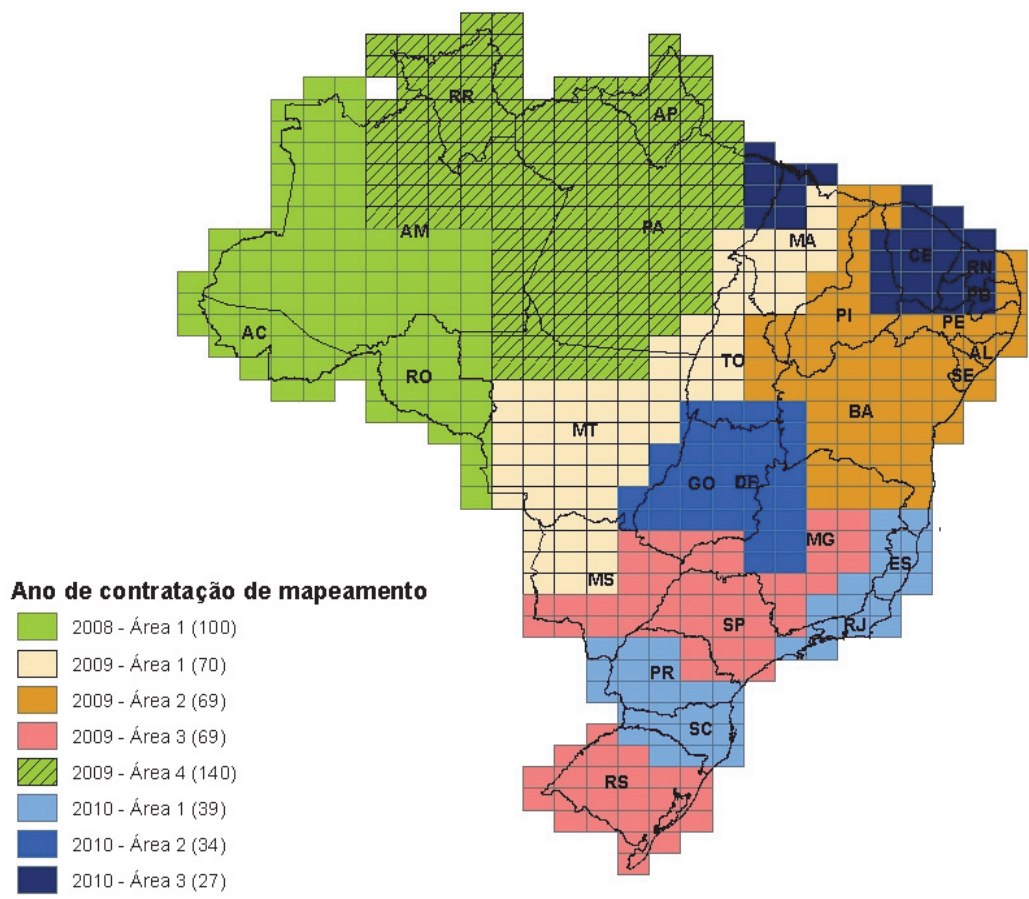

Figura 1. Mapa do Brasil com 548 folhas divididas em blocos de trabalho.

\section{Descrição do Projeto}

A implementação e a manutenção de uma base cartográfica nacional objetiva dotar o país do conhecimento físico e geométrico dos elementos componentes do território nacional. Esse conhecimento só é fornecido pelo mapeamento de referência (terrestre, náutico e aeronáutico), com a adoção de um referencial geodésico único, 
bem como a articulação dos diversos agentes de produção de forma a orientar os trabalhos, os recursos e a disseminação das informações.

O projeto BC250, de abrangência nacional, teve seu início no ano de 2007, com a elaboração das primeiras especificações técnicas e o início das atividades de mapeamento a partir de 2008. Surgiu com o propósito de prover a sociedade brasileira com um mapeamento novo, contínuo e integrado na escala 1:250.000, além de contribuir com a Infraestrutura Nacional de Dados Espaciais (INDE, 2010), que visa catalogar, integrar e possibilitar a harmonização dos dados geoespaciais existentes nas instituições do governo brasileiro. É possível encontrar mais informações sobre construção da base em outras publicações como no Simpósio Brasileiro de Sensoriamento Remoto (Reis et al., 2011) e na revista InfoGNSS (Xavier et al., 2011). Com a INDE, os dados geoespaciais, podem ser disponibilizados, localizados, explorados e acessados, para os mais diversos usos, por qualquer pessoa que tenha acesso à Internet, através do portal denominado SIG Brasil.

As feições cartográficas representadas, oriundas tanto do mapeamento novo quanto da atualização do mapeamento antigo, seguiram o padrão da Especificação Técnica para a Estruturação de Dados Geoespaciais Vetoriais (ET-EDGV), desenvolvido pelo Comitê de Estruturação da Mapoteca Nacional Digital (CEMND) que faz parte da CONCAR. Esse novo padrão de modelagem e armazenamento de dados vetoriais facilita o compartilhamento, a interoperabilidade e a racionalização de recursos entre os produtores e usuários de dados e informações (CONCAR, 2007). No processo de construção da base nem todas as classes e categorias da ET-EDGV foram contempladas, devido as restrições imposta pela resolução espacial das imagens utilizadas no projeto. A base conta hoje com 7 (sete) das 13 (treze) categorias existentes na ET-EDGV.

O levantamento e as atualizações das feições cartográficas, como sistema de transporte, hidrografia, localidades, estrutura econômica, energia e comunicação, abastecimento e saneamento e limites tiveram como base as cenas dos sensores Landsat/TM-ETM com resolução espacial de 30m, adquiridas após 2006. O sensor foi adotado por causa da sua abrangência, pois o mesmo possui cenas que cobrem todo o território nacional e estão disponíveis gratuitamente na Internet. No caso de áreas onde a cobertura de nuvens não atendia à especificação, ou seja, ultrapassavam $20 \%$ da imagem, foram utilizados outros sensores (como por exemplo o CBERS-CCD). Dados oriundos do mapeamento existente e produzidos por órgãos setoriais parceiros, como o Ministério da Aeronáutica, Ministério do Meio Ambiente, DNIT, entre outros, foram incorporados à base cartográfica utilizando-se a técnica de compilação.

As cenas foram ortorretificadas com pontos levantados em campo, onde o parâmetro adotado na especificação do projeto era de que $90 \%$ dos pontos coletados, garantissem que a base cartográfica teria uma precisão melhor ou igual a $125 \mathrm{~m}$ 


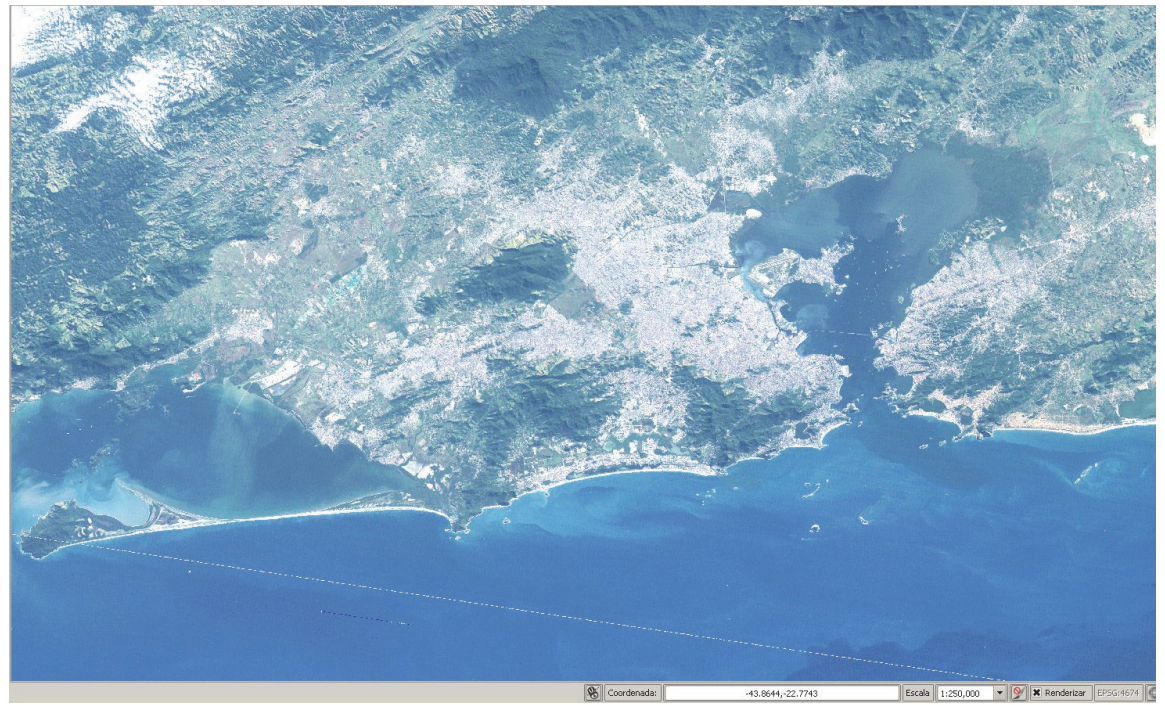

Figura 2. Cena Landsat 217/76 de 2009, ortorretificada, cobrindo o município do Rio de Janeiro.

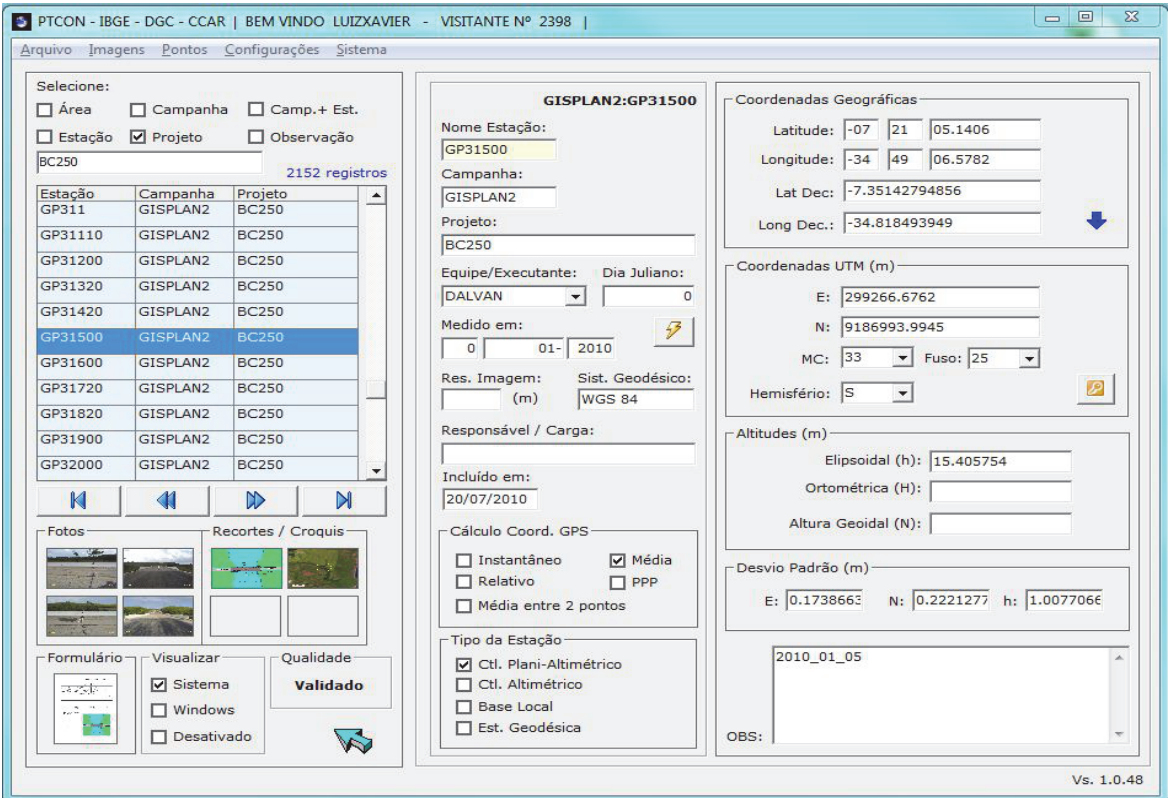

Figura 3. Interface de acesso ao banco de dados de pontos de apoio de campo. 


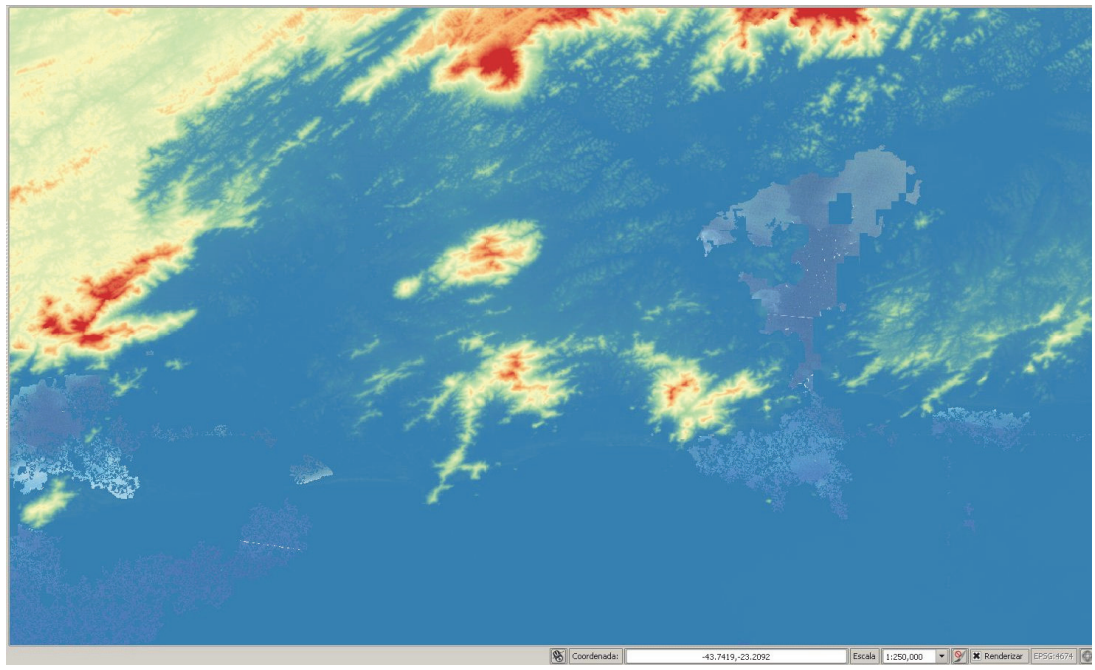

Figura 4. Modelo Digital de Elevação (MDE) SRTM cobrindo a área do Rio de Janeiro.

Base Cartográfica Contínua do Brasil na escala 1:250.000

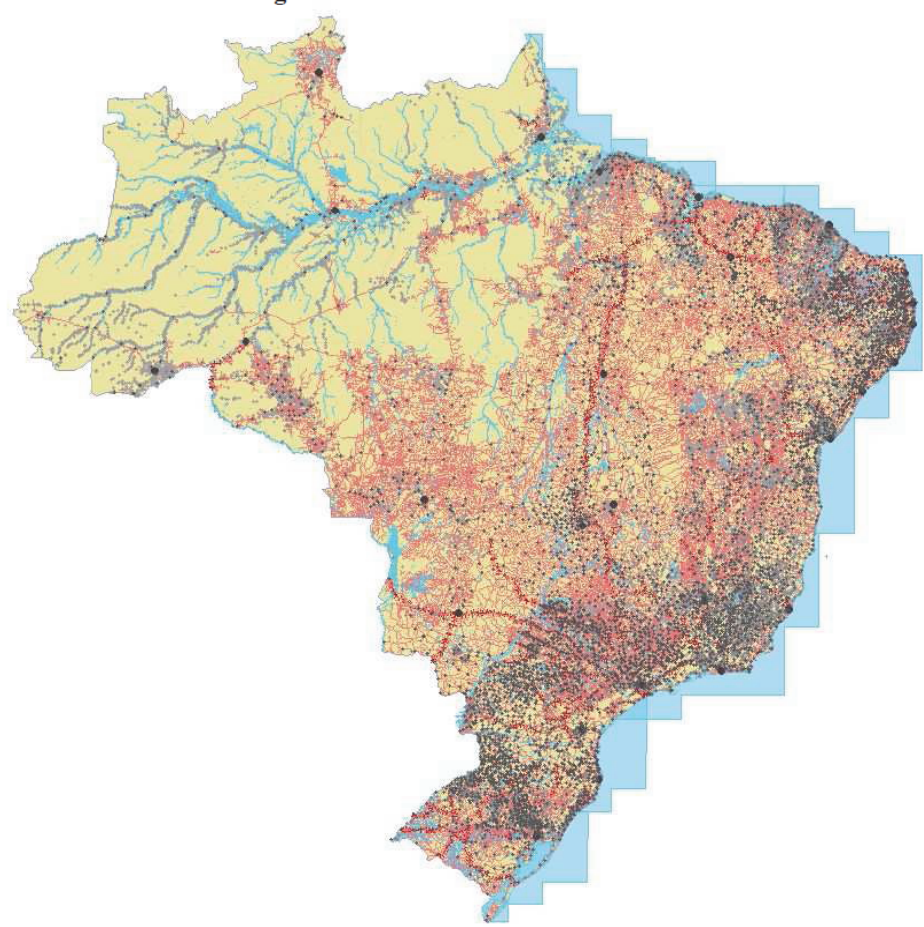

Figura 5. Base cartográfica contínua na escala 1:250.000. 
na planimetria e de $50 \mathrm{~m}$ na altimetria, valores que estão de acordo com o Padrão de Exatidão Cartográfica na categoria A (PEC A). O PEC foi instituído no decreto número 89.817 de 20 de Junho de 1984, que determina os parâmetros da cartografia brasileira. Segundo o artigo $8^{\circ}$ deste decreto, as cartas topográficas quanto à sua exatidão/classificação devem atender aos valores especificados e obedecendo os seguintes critérios para cada escala do mapeamento, segundo a Tabela 2 abaixo:

Tabela 2

Valores de PEC e EP altimétricos nas principais escalas de mapeamento sistemático brasileiro

\begin{tabular}{ccccccccc}
\hline \multirow{2}{*}{ Escala } & Eqüidistância $(m)$ & \multicolumn{7}{c}{ Classe } \\
\cline { 3 - 9 } & & \multicolumn{2}{c}{$A$} & \multicolumn{2}{c}{$B$} & \multicolumn{2}{c}{$C$} \\
\cline { 3 - 9 } & & PEC & EP & PEC & EP & PEC & EP \\
\hline $1: 250.000$ & 100 & 50 & 33,4 & 60 & 40 & 75 & 50 \\
$1: 100.000$ & 50 & 25 & 16,7 & 30 & 20 & 37,5 & 25 \\
& 40 & 20 & 13,4 & 24 & 16 & 30 & 20 \\
$1: 50.000$ & 20 & 10 & 6,7 & 12 & 8 & 15 & 10 \\
$1: 25.000$ & 10 & 5 & 3,4 & 6 & 4 & 7,5 & 5 \\
\hline
\end{tabular}

Os pontos coletados em campo para ortorretificação das imagens utlizadas no projeto foram armazenados em um banco de dados, cuja interface de acesso que está em desenvolvimento é mostrada na Figura 3 e futuramente esse mesmo banco será disponibilizado para público na página do IBGE na Internet. O procedimento para controle de qualidade e coleta dos pontos de campo podem ser acompanahdos no manual técnico de geociências número 12 do IBGE (IBGE, 2011). As coordenadas planimétricas estão referenciadas ao sistema geodésico de referência SIRGAS2000 e as altitudes dos pontos referem-se ao datum vertical de Imbituba.

Os Modelos Digitais de Elevação (MDEs) do SRTM, com resolução espacial de $90 \mathrm{~m}$ (Figura 4), foram utilizados na ortorretificação das imagens. Cabe ressaltar que, Barros (2006) e Santos (2005) atestam a compatibilidade dos produtos gerados pela SRTM para a escala de 1:250.000. Assim como as imagens Landsat, os produtos SRTM foram utilizados por terem cobertura nacional e estarem disponíveis gratuitamente na internet.

\section{Resultados e produtos}

Com a conclusão da etapa de aquisição feições vetoriais em 2011, o IBGE conseguiu produzir o mapeamento do Brasil dividido por blocos, na escala 1:250.000 conforme Figura 1 supracitada. A partir do ano de 2012, teve início a segunda etapa no 
desenvolvimento do projeto, onde o objetivo era a integração destes blocos em uma base única, estruturada conforme modelo da ET-EDGV. A integração da base foi realizada com ajuda de diversos fluxos automatizados, desenvolvidos para edição, validação e integração dos onze blocos de mapeamento, originando a primeira versão da base cartográfica contínua do Brasil na escala 1:250.000, em sua versão integrada, conforme Figura 5.

O projeto da $\mathrm{BC} 250$ tem como principais produtos disponibilizados na Internet:

- Cenas ortorretificadas - total de 379 cenas Landsat e 4 cenas CBERS.

- Base cartográfica vetorial contínua, estruturada conforme a ET-EDGV, juntamente com os metadados correspondentes.

\section{Visão de futuro para a base cartográfica contínua na escala de 1:250.000}

Atualmente, manter atualizada a base cartográfica contínua na escala de 1:250.000 de todo o território nacional através de um Programa de Atualização Permanente (PAP) passa a ser a atividade da Coordenação de Cartografia (CCAR) do IBGE.

O IBGE através da CCAR empenha-se para que a $\mathrm{BC} 250$ seja a base cartográfica de referência para os planejamentos regionais e mapeamentos temáticos de todo o território nacional. Para que isso se torne realidade algumas ações já estão em andamento.

Hoje, a BC250 conta com um acervo de imagens RapidEye, que possui uma resolução espacial original de 5.8 metros, que foram reamostradas para 5 metros ao serem adquiridas pelo Governo Federal para uso nas instituições públicas de governo. São dois recobrimentos do território, com imagens que abrangem $96 \%$ e 98\% do território nacional, e têm como anos-base 2011/2012 e 2012/2013, respectivamente.

Está em curso o desenvolvimento de uma ferramenta que estará disponível na página do IBGE na Internet para facilitar a interface/navegação com todos os usuários. Ainda nesta etapa do PAP estão previstas também campanhas de campo denominadas de reambulação. A reambulação é a técnica que consiste em levantamentos em campo da denominação e classificação de acidentes naturais e artificiais (hipsográficos, hidrográficos, do sistema de transporte, de localidades, obras de engenharia em geral e marcos de divisas dentre outros), tendo como base insumos que podem ser: fotografias aéreas, restituições fotogramétricas da área, imagens de radar, de satélite, mosaicos e ortofotos. Durante as atividades de reambulação serão levantados, identificados e classificados os elementos do terreno, a serem cartografados, para complementação das informações de toponímia da base de dados vetoriais.

$\mathrm{O}$ tratamento, a normalização e a carga oriunda dos topônimos da $\mathrm{BC} 250$, de todo território nacional, dos nomes geográficos para o Banco de Nomes Geográficos do Brasil (BNGB), outro produto do IBGE. 
Dentre as principais atividades que serão desenvolvidas no PAP, destacam-se:

- Edição e validação da base vetorial integrada;

- Possibilitar o acesso on line para as unidades descentralizadas do IBGE, para consulta e edição da base;

- Uso de MDEs para extração das informações altimétricas do projeto;

- Complementação das categorias e feições da EDGV correspondentes à escala de mapeamento;

- Complementação de informações através de consulta a outros bancos de dados e a outros insumos cartográficos como as rodovias do DNIT, as Unidades de Conservação do IBAMA, etc;

- Atualização das folhas com as informações reambuladas;

- Banco de dados dos pontos de apoio de campo e respectivas coordenadas planilatimétricas, determinadas com equipamentos GPS de navegação;

- Mosaicos ortorretificados das cenas por recorte das folhas 1:1.000.000 -46 mosaicos;

- Mosaicos dos Modelos Digitais de Elevação por recorte de folhas 1:1.000.000 - 46 mosaicos;

- Utilização de fluxos automatizados para validações geométricas, topológicas e homogeneização de nomes geográficos — 78 fluxos;

- Utilização da base cartográfica para a elaboração de mapas estaduais.

\section{Considerações finais}

Através da implementação de um ambiente operativo com banco de dados/SIG, com dados integrados e estruturados, é possível agilizar a confecção de documentos cartográficos, utilizados como referência básica para o planejamento ambiental, o mapeamento de uso e cobertura do solo, o reflorestamento, as políticas públicas sociais e de defesa, o uso e conservação do potencial hídrico, a infraestrutura básica, a proteção e conservação ambiental, o estudo das potencialidades minerais, o planejamento dos sistemas de transportes rodoviários, hidroviários e ferroviários, dentre outros.

\section{Referências Bibliográficas}

Barros, R.S. de (2006). "Avaliação da Altimetria de Modelos Digitais de Elevação Obtidos a Partir de Sensores Orbitais [Rio de Janeiro]", D.Sc., Geografia, Tese, Universidade Federal do Rio de Janeiro, IGEO/UFRJ, XIX, 172 pp.

Brasil. Decreto $\mathrm{n}^{\circ}$ 89.817, de 20 de junho de 1984, disponível em: $<$ http://www.planalto.gov.br/ccivil_03/decreto/1980-1989/D89817.htm>, acessado em junho de 2011.

CONCAR, Comissão Nacional de Cartografia. "Especificações Técnicas para Estruturação de Dados Geoespaciais Digitais Vetoriais", Versão 2.0, 2007. Disponí- 
vel no portal da CONCAR, disponível em: <http://www.concar.gov.br/>, acesso em: 09 de novembro de 2010.

Delou, A.L. de A. (2006). "Estruturação de Bases Cartográficas para SIG: Um estudo comparativo dos modelos do IBGE e da DSG", dissertação, Universidade Estadual do Rio de Janeiro, FEN/UERJ, 276 pp.

IBGE (2003). Mapa Índice Digital, Rio de Janeiro, IBGE, CD-Rom.

- (2011). "Manual de procedimentos técnicos para fiscalização, controle de qualidade e validação da base cartográfica contínua na escala 1:250.000", Manuais Técnicos em Geociências, no. 12.

INDE, Infraestrutura Nacional de Dados Espaciais, disponível em: $<$ http://www.inde.gov.br/>, acesso em 09 de novembro de 2010.

Maranhão, M.R.A. (2005). "Modelo de seleção de áreas para atualização do Mapeamento Sistemático baseado em lógica nebulosa", XIII, Área de Concentração Geomática, Dissertação, Universidade do Estado do Rio de Janeiro (UERJ), 102 pp.

Reis, R.B.; Medeiros, D.R.; Lopes, M.; Cruz, C.B.M. e Barros R.S. de (2005). "O uso de produtos de Sensoriamento Remoto gratuitos na representação do Relevo - um potencial para a educação", Anais XII Simpósio Brasileiro de Sensoriamento Remoto, Goiânia, Brasil, 16-21 abril, INPE, p. 1337-1344.

Reis, R.B.; Xavier, L.A.; Krumbiegel, M.; Barbosa, F.O.; Pereira, P.B.M., Azevedo, J.B. et al. (2011). "Mapeamento da base contínua do Brasil na escala 1:250.000 com auxílio de sensores orbitais - Projeto BC250", XII Simpósio Brasileiro de Sensoriamento Remoto, Anais do XV Simpósio Brasileiro de Sensoriamento Remoto, Curitiba, Brasil INPE, pp. 2452-2459.

Santos, P.R. a dos (2005). "Avaliação da Precisão Vertical dos Modelos SRTM em Diferentes Escalas: Um estudo de caso na Amazônia”, dissertação, Rio de Janeiro, Instituto Militar de Engenharia, 116 pp.

Santos, P.R. a dos e Oliveira, L.M.T. de (2003). "Processamento digital de imagens realizado no IBGE", Anais XI do Simpósio Brasileiro de Sensoriamento Remoto, Belo Horizonte, Brasil, INPE, pp. 2123-2128.

Shutle Radar Topography Mission (SRTM). Disponível em: http://srtm.csi.cgiar.org/SELECTION/inputCoord.asp/, acesso em 09 de novembro de 2010.

Xavier, L.A.; Reis, R.B.; Krumbiegel M.; Barbosa, F.O.; Pereira, P. e Azevedo, J. (2011). "Mapear o Brasil na escala 1:250.000, um desafio. InfoGNSS", A revista do profissional de Geomática, no. 36, pp. 44-46. 